\title{
Software Testing and Verification in Climate Model Development
}

\author{
Thomas L. Clune NASA Goddard Space Flight Center \\ Richard B. Rood University of Michigan Atmospheric, Oceanic and Space Sciences
}

\begin{abstract}
:
Over the past 30 years most climate models have grown from relatively simple representations of a few atmospheric processes to a complex multi-disciplinary system. Computer infrastructure over that period has gone from punch card mainframes to modern parallel clusters. Model implementations have become complex, brittle, and increasingly difficult to extend and maintain. Existing verification processes for model implementations rely almost exclusively upon some combination of detailed analysis of output from full climate simulations and system-level regression tests. In additional to being quite costly in terms of developer time and computing resources, these testing methodologies are limited in terms of the types of defects that can be detected, isolated and diagnosed. Mitigating these weaknesses of coarse-grained testing with finer-grained "unit" tests has been perceived as cumbersome and counter-productive. In the commercial software sector, recent advances in tools and methodology have led to a renaissance for systematic fine-grained testing. We discuss the availability of analogous tools for scientific software and examine benefits that similar testing methodologies could bring to climate modeling software. We describe the unique challenges faced when testing complex numerical algorithms and suggest techniques to minimize and/or eliminate the difficulties.
\end{abstract}

\section{Introduction}

Testing, verification, evaluation and validation are vital aspects of the construction of climate models. However, these processes are ingrained into the cultures of modeling centers and are often not specifically recognized. ${ }^{[1]}$ Here, we distinguish validation and verification as in Post and Kendall ${ }^{[2]}$ where validation refers to comparison with observations and verification refers to comparison with analytic test cases and computational products. Testing and evaluation are more generic and will be defined more concretely below.

From a software-focused perspective, our community is dominated by software developed by scientists as opposed to software engineers. ${ }^{[3]}$ The community invests heavily in system-level testing which exercises most portions of the underlying code base with realistic inputs. These system-level tests verify that multiple configurations of the model run stably for modest time intervals as well as certain key invariants such as parallel reproducibility and checkpoint restart. Contrariwise, the routine application of fine-grained, low-level tests (unit tests) is nearly nonexistent. Notable exceptions are limited to "infrastructure" software such as that of the Earth System Modeling Framework (ESMF). Infrastructure is typified by its role in supporting data organization and movement such as for parallelism, $1 / \mathrm{O}$, and transferring information from, for example, ocean grid to atmosphere grid. ${ }^{[4]}$

The lack of use of unit tests in climate model development is in stark contrast to the practices of commercially developed software. Many reasons can be posited for this situation, but we argue 
that the fundamental reasons are a lack of awareness of the benefits of pervasive unit testing as well as strong misconceptions about the nature and difficulty of implementing unit tests. We argue here that analysis of the model evaluation process reveals the elements of the ingrained culture of testing and evaluation. This allows the definition of a more comprehensive approach to software testing. Among the benefits of a more formal approach to testing are improved software quality, reliability and productivity of model developers.

\section{Climate Model Practice}

In simplistic terms the process of model development can be conceived as a 2-phase cycle that alternates between development and evaluation. During development the programmer extends the capabilities of the model by producing new code. Then, during evaluation the programmer seeks to verify whether those changes achieve the desired results without breaking other aspects of the implementation. Different scales of code changes warrant different types of verification, and all successful verifications are tentative as later verifications may reveal flaws.

A key observation about the above process is that developers naturally scale the magnitude of the verification phase to match computational resources and time required for the process. For example, if the verification technique requires lengthy simulations, then developers are unlikely to perform verification upon each new line of source code. Rather, they are likely to spend one or more days programming prior to submitting a verification run. Likewise, if the verification process is merely to ensure no compilation errors, developers may perform the verification frequently throughout the day.

Our focus is ultimately on model evaluation processes that are not comparisons with observations, i.e. validation. However, it is useful to disentangle testing, verification and validation more thoroughly. Validation is a controversial issue in climate modeling centers. Indeed, Oreskes at al. ${ }^{[5]}$ argued that formal validation of geophysical models of complex natural systems is "impossible." More recent papers studying the philosophy and social science of the culture of climate and weather modeling suggest that the extensive comparison with observations and the extensive review of climate assessments do stand as validation. ${ }^{6]}$

Because of formal programmatic requirements the Data Assimilation Office (DAO now the Global Modeling and Assimilation Office) at NASA's Goddard Space Flight Center was required to produce a formal validation plan. ${ }^{[7]}$ In the DAO validation plan the strategy was taken to define a quantitative baseline of model performance for a set of geophysical phenomena. These phenomena were broadly studied and simulated well enough that they described a credibility threshold for system performance. Important aspects of this validation approach were that it is defined by a specific application suite, formally separated validation from development, and relied on both quantitative and qualitative analysis.

In this paper we are focused on the testing and evaluation that are specific to the software itself. That is, testing and evaluation that should be completed prior to the submission of the system for validation. Numerous studies have demonstrated that the cost of fixing a software defect is substantially less if detected early. ${ }^{8}$ A primary verification mechanism is a short-duration run of the full modeling system. Even when such tests can be performed in a matter of minutes, they are 
often too crude to detect all but the most basic sorts of errors. Defects, therefore, fester until more thorough runs are performed and detailed analyses of the results are examined. When possible, finer-grained tests which directly verify the specific changes being attempted not only execute faster, but also have a higher probability of detecting undesired behavior earlier. Professional software engineers have recognized these characteristics of software development and have generally moved towards development processes which provide very rapid cycling between coding and verification.

\section{Software Testing}

Software testing can be applied at various levels within a climate model. Coarser-grained tests, e.g. those that encompass large portions of the implementation, can be useful for detecting the existence of defects, but are generally limited in their ability to isolate specific causes. In contrast, fine-grained tests are better at isolating and diagnosing specific defects, but the number of tests necessary to cover an entire application may be prohibitively expensive. Coarse-grained tests of modern parallel climate models may also require substantial computational resources for their application, whereas with appropriate care, fine-grained tests can be implemented to require only minimal computational resources. In practice, coarse-grained tests are applied nightly or weekly, while fine-grained tests are exercised more frequently to support ongoing development. Projects must consider the relative costs and benefits of each category of testing when formulating a testing plan. Below we define a categorization of tests.

\section{System Tests}

The coarsest level of testing is system testing, in that individual tests exercise all or nearly all of the underlying implementation. Most system tests are in the form of regression tests -- i.e. the tests aim to reveal errors that have been introduced to existing functionality. Regression tests commonly used in climate modeling seek to answer questions such as:

- Do the primary model configurations run to completion?

- Are the results independent of parallel decomposition?

- Does the process of checkpoint/restart alter the results?

- Has the computational performance significantly changed?

Although the process of regression testing is similar to studying the consequences of scientific changes to the model, the distinction is important. A regression test is objective and can be automated. ${ }^{[9]} \mathrm{A}$ scientific change involves tradeoffs on subtle qualities of a model and requires a variety of investigations into the model behavior.

One of the impediments to system testing in most, if not all, climate models is the large number of configurations that could be tested. For example, models can be configured with and without components such as an ocean, atmospheric chemistry, and aerosols, each of which may have alternate implementations. The number of potentially supported combinations grows exponentially with the number of components and can limit the extent to which the system is thoroughly tested. 
Despite the difficulties induced by the proliferation of model configurations, system level testing is where climate models have the greatest investment and maturity. For example, GISS ModelE

${ }^{[10]}$ is developed with continuous integration, which allows nightly automated tests which checkout the latest development branch and compile and execute $\sim 10$ model configurations under a variety of parallel decompositions. Alerts are emailed if a configuration fails to compile or execute, or if the results are not strongly reproducible. Even when the test reports are ignored in real time, experience shows that these tests often bound the dates on which a defect was introduced and greatly reduce the effort to identify and fix the problem. Other climate teams typically have longer integration cycles, but can compensate by more intensive testing at those boundaries.

Integration testing

Integration testing is the testing of software aggregates and is intermediate between system testing and fine-grained unit testing. Although there is much to be said about integration testing, the distinction is of little consequence to the arguments in this paper.

\section{Unit testing}

Unit tests are the finest granularity of testing and are used to verify low level behaviors of an implementation. Generally a unit test exercises a single unit (function or subroutine) by comparing the output generated from a set of synthetic input values against the corresponding output values. Some special unit testing concerns that arise for climate modeling include parallelism, checkpoint/restart, and numerical computations. Automated fine-grained testing is quite limited in current climate models, with the few counter examples generally associated with infrastructure layers.

\section{Unit testing frameworks}

Until quite recently, software developers dreaded the process of creating unit tests. The level of effort for producing tests was perceived to be high and a distraction from producing "real" software. Two innovations have influenced this attitude. The first was the creation of languagespecific unit testing frameworks, e.g. JUnit, which reduce the difficulty of creating and executing tests and reporting test results. The second innovation was the adoption of test-driven development ${ }^{[1]]}$ in which the conventional software development process is reversed with developers creating tests prior to the software to be tested. The change in developer perception of the role of unit tests is perhaps best typified by this quotation from Michael Feathers: "The main thing that distinguishes legacy code from non-legacy code is tests, or rather a lack of tests." [12] The implication is that the existence of a sufficiently robust suite of tests completely alters the experience, productivity, and overall risk when working with a software system.

Spurred on by the success of JUnit, unit testing frameworks have been created for most modern programming languages. There are at least three independent frameworks for Fortran, including pFUnit, which also supports testing parallel implementations based upon MPI. Although these frameworks vary from language to language, their basic architectures are quite similar. Each framework provides a suite of assertion routines, which can be used by the developer to express 
the expected state of output variables after a call to the routine being tested. When an assertion fails to hold, the framework logs the name of the test that fails along with the location of the assertion and any accompanying user-provided messages for that assertion.

\section{Test-Driven Development (TDD)}

Test-driven development (TDD) is a major element of agile development processes and consists of a short development cycle that alternates between development of tests and development of code which enables the tests to pass. First, the developer creates or extends a test which then fails because the necessary functionality has not yet been created. Then the developer produces code just sufficient to pass the test. Finally, the developer cleans up with an emphasis on removing any incidental redundancy. The process encourages progress in the form of rapid, incremental steps and is made practical through unit testing frameworks.

To better understand the TDD workflow and its application in the context of numerical software, consider the process of developing a simple 1D linear interpolation procedure. The first step is to create a test. Usially the first test is extremely simple and is primarily intended to specify the desired interface for the procedure to be implemented, e.g.

assert $(0==$ interpolate $(x=[1,2], y=[0,0]$, at_ $x=1))$

With this test in place, we begin to implement the interpolate procedure itself, but only so far as to enable successful compilation and execution of the test. A trivial implementation that returns zero will suffice. For the next test we choose data that have a simple linear relationship:

$\operatorname{assert}(1==$ interpolate $(x=[0,2], y=[0,2]$, at_ $x=1))$

This test will already compile and execute, but it will fail due to the overly simplistic implementation after the first test. We proceed by extending the implementation to pass both tests. Additional tests could then be to check that the behavior is correct when there are multiple intervals in the data, or when the interpolation is outside the domain, the data are degenerate, etc. After each test, the interpolate procedure is extended to ensure that all tests pass. For many it will be initially counterintuitive to proceed in such minute steps, but with practice these steps happen very quickly and lead to steady predictable development of complex features.

The practitioners of TDD tout many benefits. Chief among these is the improvement in overall developer productivity despite the substantial increase in the total number of lines of code for a given piece of functionality. The improved productivity stems from the comprehensive test coverage which in turn leads to far fewer fixes to defects later. Tests under TDD are continuously exercised and can, therefore, serve as a form of maintainable documentation. Developers using TDD may also be more productive due to reduced stress and improved confidence that arise from the immediate feedback as functionality is extended. Finally, TDD leads to higher quality implementations. This somewhat surprising claim stems from the observation that software that is designed to be testable tends to be comprised of smaller procedures having shorter argument lists. 


\section{Barriers to Testing}

In this section we explore the barriers, both technical and cultural, to more pervasive adoption of testing methodologies in climate models.

Scientists often perceive testing as an attempt to execute a model, a component, or an algorithm with maximal fidelity. Their instinct is to use representative grid resolutions, realistic input values, etc. The art of software testing is to use synthetic input values and small grids that exercise specific lines of code to produce output values that can be verified by inspection. Highresolution grids serve no purpose other than to slow the execution of tests and require more memory. The use of realistic input values is appropriate only to avoid branches that are unsupported by the implementation, or when independent realistic output values are available. Working with synthetic inputs does require some care to ensure that important cases are covered. For example, frequent programming errors such as index permutations and wrong offsets can be detected by imposing a spatial dependence on test inputs.

The largest technical barrier to unit testing in climate modeling software is the legacy nature of the code base. Large ( $>1000$ sloc) procedures that rely heavily on the use of global variables are difficult to characterize in the form of a unit test. A possible test might be to store representative input and output data of a procedure in an external file. Tests could then be developed which exercise the procedure with the stored data and notify if any output values have changed. Such tests would be of limited value other than for the purposes of refactoring.

Teams that desire to enhance software testing mitigate the problem of legacy software by limiting modification of existing routines. Rather than "wedging" new code into a large procedure, they develop a new testable subroutine. In the legacy procedure they insert, only, a call to the new routine. Groups also look for opportunities to extract small testable routines from large legacy procedures.

Beyond the burdens of the legacy code base, numerical algorithms present difficulties that are absent in many other categories of software. The most basic of these arise from numerical errors that originate from both truncation and round off of floating point operations. Such errors are problematic because the corresponding tests must not only specify expected values for output parameters, but also provide tolerances for acceptable departures from those expected values. If the tolerances are too tight, then tests will appear to fail despite producing acceptable results, whereas if tolerances are too loose, then tests lose value as they fail to detect some errors. In most cases, developers do not have suitable a priori values for such tolerances, even when the asymptotic form of the truncation error for an algorithm is known.

Fortunately, the difficulty of specifying suitable tolerances is not as severe in practice as the above discussion indicates. First, note that the tests are verifying the implementation - not the asymptotic form of the algorithm. When an algorithm is broken down into sufficiently small steps, the resulting pieces are often amenable to simple, even trivial, error analysis. Complicated error bounds are largely a consequence of the how errors compound through subsequent stages of a calculation, and from the testing perspective, this compounding can be avoided by examining each step independently. 
An important concern of climate models is whether small changes to an implementation may result in a different basin of attraction for the trajectories and hence a different climate. The chaotic nature of the underlying dynamics effectively limits the ability of unit tests to constrain the system in this regard, and only long control runs can protect against undesirable changes. Likewise, so long as all "local" tests pass, long control runs cannot identify any particular aspect of the implementation that is responsible for an incorrect result, though the most recent change will often be the assumed offender. Over time, researchers identify additional constraints (and thus unit tests) that decrease the frequency of incorrectly altering the simulated climate, but verification will always require control runs.

Another major objection to testing of some numerical algorithms is the general lack of known, analytic solutions for realistic algorithms that could be used to derive appropriate values to use in tests. Unless an equivalent and entirely independent algorithm is available, tests of such algorithms tend to be redundant with the implementation and thus of no real value. As with the discussion of numerical errors above, this concern can largely be eliminated by decomposing algorithms into distinct, simple steps, for which synthetic test values are readily apparent. For pedagogical purposes, consider how testing would be implemented for a procedure which computes the area of a circle.

area $=$ areaofCircle(radius)

One might choose a small set of trial values for the input radius: $0,1,2$ and verify that the results are $0 ., 3.14159265,12.5663706$ respectively. These results are not obvious by inspection because mental arithmetic with $\pi$ is problematic. One could replace these literal values with expressions involving $\pi$, but that the resulting test is very nearly redundant with the algorithm being tested. However, from the software engineering perspective, there is a different option: introducing $\pi$ as a second parameter into the interface. We can then use simple values of " $\pi$ " such as 1 or 2 for testing the implementation of the procedure. One would implement the usual interface for areaOfCircle (radius) by passing a hardcoded value of $\pi$ to the procedure that accepts two arguments. This hardcoded value of $\pi$ can be tested by inspection or by trigonometric identities.

This methodology for decomposing numerical schemes may seem overly burdensome at first; however, we argue that compared to the level of effort of developing a scientific parameterization, the effort spent on expressing algorithms in this fine-grained manner would be modest, and the return might be immeasurable in the form of early detection of software defects. This approach also helps to side step the issue of providing realistic input values for complicated physical parameterizations based upon empirical parameters and curve fitting. Of course, if realistic input and output data are available, then corresponding tests should be created. More often, though, high-level tests for such parameterizations should verify the sequence of sub-steps rather than attempting to compose a full numerical comparison. This approach is more natural with abstract data types and object-oriented programming, and may require re-engineering a relatively small number of interfaces in a model.

More broadly, the technique of splitting scientific models into very small procedures to enable unit testing raises concerns about computational efficiency. If algorithms are split into very small 
procedures with short parameter lists, then the overhead cost in terms of performance in a real code could be substantial. This difficulty can be overcome via bootstrapping. Namely, an optimized implementation (large monolithic optimized procedure) can be tested against the slower fine-grained implementation that is in turn covered by simple, clean unit-tests. Although this technique introduces yet more overhead to the development process, the fine-grained implementation can itself be a very useful reference for attempts at further optimizing an algorithm.

\section{Applications of Test-Driven Development}

In the Software Integration and Visualization Office (SIVO) at the NASA Goddard Space Flight Center, we have aggressively applied TDD to several development projects including two that are directly relevant to scientific modeling. The first of these was to develop a parallel application, GTRAJ, which calculates the trajectories of billions of air parcels in the atmosphere. The other was to develop a parallel implementation, SNOWFAKE, of a numerical model ${ }^{[13]}$ that simulates the growth of virtual snowflakes. The success of these projects demonstrates that unit testing in general, and TDD in particular, can be applied in the modeling arena. These projects also revealed the challenges of repressing established programming habits and methodology on the part of senior scientific programmers.

GTRAJ is a complete rewrite of a legacy application, the Goddard Trajectory Model ${ }^{[14]}$ originally developed in IDL. To achieve portability and scalability, SIVO applied TDD to rewrite the application in $\mathrm{C}++$ and MPI using a team of senior scientific programmers that were relatively inexperienced with unit testing and TDD. The primary numerical algorithms used in GTRAJ are numerical interpolation of gridded meteorological data, and temporal integration of the interpolated flow field experienced by a given parcel of air. The original model was primarily tested by measuring the discrepancy in time reversal of a five-day integration. Although seemingly stringent, the requirement of time-reversibility is unable to detect many types of errors. During the rewrite the new fine-grained tests revealed two important errors in the original. First, the binning algorithm which selects which grid points should be used for interpolation was incorrect in some regions with sharp changes in the pressure heights. The "interpolations" would then become extrapolations and occasionally extreme inaccuracies would result. The correction would have been difficult in the original monolithic implementation, but was straightforward in the version developed under TDD. The second error detected by unit testing in the development of GTRAJ was the behavior of Runge-Kutta (RK) when parcels pass near the poles in a latitudelongitude grid. Examination of the large errors for such trajectories using synthetic test data, led to the realization that the usual RK algorithm must be reformulated for curvilinear coordinate systems. With this correction, the accuracy of trajectories that pass near the poles was improved by orders of magnitude which in turn permitted substantially larger time steps and faster execution of the application.

At several points in the creation of GTRAJ, the primary developers were challenged by the process of following TDD. The temptation to immediately begin implementing an algorithm was difficult to resist, especially early in the project. Unfortunately, the pressure to skip tests is typically highest when the tests do the most good either because the design requires further thought, or the expected behavior is harder to specify. Scientific programmers also struggled 
with a tendency to use realistic input values in tests rather than "enlightening" values, but rapidly improved.

In contrast to GTRAJ, the initial serial implementation of SNOWFAKE was developed by a single developer who was experienced in using TDD for components of model infrastructure. The project benefited from the precise mathematical specification of the model in the reference paper which was translated into a set of basic tests. The initial implementation was completed in approximately 12 hours of development time and was comprised of approximately 700 lines of source code and 800 lines of tests. The application ran to completion on the first try and produced simulated snowflakes in excellent agreement with the reference paper. The simple "micro" debugging at each stage of TDD apparently eliminated the substantial debugging typically experienced during the integration phase of programs of even less complexity. Other encouraging statistics are that procedures averaged under 12 lines and less than 2 parameters (arguments). As one might expect, the initial implementation was suboptimal in terms of computational performance, but optimized variants of key algorithms were then easily created and verified by tests that compared optimized results against the slower baseline implementations. For example, one significant cache-based optimization was from fusing the sub steps associated with vapor diffusion. The test for the fused procedure was simply to compare the results of the fused procedure with a sequential execution of each original sub step. Both implementations and corresponding tests were retained.

\section{Conclusions}

Confidence in the scientific predictions of climate models is contingent upon confidence in the underlying implementations of those models. In this paper we have advocated that current software development practices are inadequate for gaining such trust, and that the increased use of automation and systematic fine-grained testing of model implementations would be a major improvement in this regard. Further, such testing has been shown to provide a net improvement to developer productivity in other communities through a number of indirect mechanisms.

Our experience with two applications suggests that this testing methodology is applicable to climate modeling, but more relevant examples are needed. Changing established processes in any community is difficult, and scientists are understandably skeptical about the costs and benefits of our approach. In the near future we intend to apply test-driven development in the creation of a parameterized model component from scratch. The early phases of the development of such a component are the ideal time to express the various physical requirements and constraints as unit-tests, and the results should serve as a strong demonstration of the potential of this technique.

Many technical difficulties must be overcome before unit testing can become pervasive within climate models. Chief among these is the extreme difficulty of introducing fine-grained tests into the procedures typical of legacy science code. In many cases, this issue can by sidestepped by implementing changes and extensions to models as new modules. This is in contrast with the common practice of "wedging" changes directly into old procedures and ultimately compounding the legacy burden. Fixes to software bugs are a particularly important case to follow this approach, as the constructed test serves as protection against re-introduction of the 
defect. In the long term more powerful tools must be developed which allow developers to efficiently extract disjoint bits of functionality from the legacy layer. In other programming languages, especially Java, new generations of powerful software developer tools have been shown to be quite effective for such purposes. Photran ${ }^{[15]}$ is an attempt to introduce some of these capabilities for Fortran, but greater investments should be made to bring appropriate tools to a state of maturity suitable for routine application to climate models.

\footnotetext{
${ }^{1} \mathrm{~S}$. Shackley, "Epistemic lifestyles in climate change modeling," in Changing the Atmosphere, C. C. Miller and P. N. Edwards, Eds. Cambridge, MA: The MIT Press, 2001, pp 107-133.

${ }^{2}$ D. E. Post and R. P. Kendall, "Software project management and quality engineering practices for complex, coupled multiphysics, massively parallel computational simulations: Lessons leamed from ASCI," The International Journal of High Performance Computing Applications, vol. 18, pp. 399-416, Winter 2004.

${ }^{3}$ S. M. Easterbrook and T. C. Johns, "Engineering the software for understanding climate change," Computing in Science and Engineering, vol. 11, pp. 64-74, 2009.

${ }^{4}$ C. Hill. C. DeLuca, V. Balaji, M. Suarez, and A. da Silva, "Architecture of the Earth System Modeling Framework," Computing in Science and Engineering, vol. 6, pp. 18-28, 2004.

${ }^{5}$ N. Oreskes, K. Shrader-Frechette, and K. Belitz, "Verification, validation, and confirmation of numerical models in the Earth sciences," Science, vol. 263, pp. 641-646, 4 February 1994.

${ }^{6} \mathrm{H}$. Guillemot, "Connections between simulations and observation in climate computer modeling. Scientist's practices and "bottom-up epistemology" lessons," Studies in History and Philosophy of Modern Physics, vol. 41, pp. 242-252, 2010.

${ }^{7}$ R. B. Rood and Staff. (1996, Nov.). Algorithm Theoretical Basis Document for Goddard Earth Observing System Data Assimilation System (GEOS DAS) with a Focus on Version 2. [Online]. Available: http//eospso.gsfc.nasa.gov/eos homepage/for scientists/atbd/docs/DAO/atbd-dao.pdf

${ }^{8}$ RTI. (2002, May). The Economic Impacts of Inadequate Infrastructure for Software Test. [Online]. Available: http/www.nist.gov/director/planning/upload/report02-3.pdf

${ }^{9}$ S. Vasquez, S. Murphy, and C. DeLuca. (2011, Apr.). Earth System Modeling Framework Software Developer's Guide. [Online]. Available: http:/www.earthsystemmodeling.org/documents/dev guide/

${ }^{10}$ G. A. Schmidt, R. Ruedy, J. E. Hansen et al., "Present day atmospheric simulations using GISS ModelE:

Comparison to in-situ, satellite and reanalysis data," J. Climate, vol. 19, 153-192, 2006.

$"$ K. Beck, Test-driven development: By example. Boston, MA: Addison-Wesley, 2003, pp. 240.

${ }^{12} \mathrm{M}$. Feathers, Working effectively with legacy code. Upper Saddle River, NJ: Prentice-Hall, 2004, pp. 456.

${ }^{13}$ J. Gravner and D. Griffeath, "Modeling snow-crystal growth: A three-dimensional mesoscopic approach," Phys. Rev. E., vol. 79, DOI: 10.1103/PhysRevE.79.011601,2009.

${ }^{14}$ M. R. Schoeberl and L. C. Sparling, "Trajectory Modeling," in Diagnostic Tools in Atmospheric Physics, J. C. Gille and G. Visconti Eds., Proc. Internat. School of Phys. "Enrico Fermi," vol. 124, 1995, pp. 419-430.

${ }^{15}$ photran - An integrated development environment and refactoring tool for Fortran. [Online]. Available: http:/www.eclipse.org/photran/
} 